\section{SCORE: An interactive FORTRAN program for computing case, item, and scale statistics}

\section{ENID BURROWS and SUZI SNOOK}

Plymouth State College, Plymouth, New Hampshire 03264

When analyzing scales, researchers may need individual (case) statistics, scale statistics, and item statistics for the entire scale and/or any arbitrary subscale. SCORE is an interactive program to analyze scales and/or subscales composed of items that can be scored dichotomously, that is, exactly one desired response for each item. It is assumed that the variable underlying this dichotomy is continuous, and hence the biserial correlation rather than the point biserial correlation is computed.

Input. Case responses and key are stored in a cardimage disk file distinct from SCORE. Information that is entered interactively includes: name of the file where data is stored (this feature allows analysis of successive data sets); number of items on the scale (subscale) being analyzed; number of possible choices for each item; name of the scale; and five options for specific output. In addition, SCORE utilizes a file containing normal distribution data necessary to the calculation of the biserial correlation (Magnusson, 1967).

Output. Case statistics (ordered by raw score): printout of raw data items; raw scale (subscale) score and score corrected for guessing or random response (optiona); Pearson product-moment correlation between raw score and score corrected for guessing.

Item statistics: item distractor display by quintiles, determined by raw scale scores (optional) (for a further discussion of this technique see Carry \& Wilson, 1968); item mean ( $\mathrm{p}$ value) and variance; item mean corrected for guessing; item mean adjusted for nonperformance or missing data (considering only those cases who responded); biserial correlation; nonspurious biserial correlation (between item and total scale score with the item removed) (Henrysson, 1963); percent of cases not responding to the item; Pearson product-moment correlation between item mean and item mean corrected for guessing.

Scale statistics: number of cases; mean raw score and standard deviation; mean score corrected for guessing and standard deviation; Cronbach's alpha measure of reliability; Spearman-Brown prophecy formula estimate of reliability for standard scale length of 50 questions; standard error of measurement; frequencies and histogram (optional).

Language. The program was written in FORTRAN IV, for the DEC-10 system.

Limitations. The program is presently dimensioned to handle up to 100 cases and up to 70 items. Depending upon the amount of core storage available to the user, the program dimensions may be adjusted simply by changing the DIMENSION statements at the beginning of the program and subroutines.

Acknowledgment. The program was developed at the University of New Hampshire, Durham, and computer time was made available by the institution.

Availability. A source listing, documentation, and sample run may be obtained free of charge by writing Enid Burrows, Department of Mathematics, Plymouth State College, Plymouth, New Hampshire 03264.

\section{REFERENCES}

CARRY, L. R..\& WILSON, J. W. Item analysis procedures used in the development of tests for the National Longitudinal Study of Mathematical Abilities. (NLSMA Working Paper No. 184) Stanford: School Mathematics Study Group, 1968.

HENRYSSON, S. Correction of item-total correlations in item analys is. Psychometrics, 1963, 28, 211-218.

Magnusson, D. Test theory. Reading, Mass: Addison-Wesley, 1967.

(Received for publication May 9. 1977.) 